\title{
Esthetic ceramic veneers for spaced anterior teeth utilizing the RED proportion; a case report with three years follow-up
}

\begin{abstract}
Discrepancy between a large jaw size and small teeth results in a spaced alignment of teeth. Orthodontic treatment to correct such cases will not be sufficient for esthetic results of anterior teeth. Restorative treatment may be the ideal solution to reshape small teeth in well planned proportion to fit the size of the jaw and enhance the appearance of the patient smile. This paper presents the process of restoring spaced anterior teeth with minimally prepared ceramic veneers using the RED proportion.
\end{abstract}

Keywords: spaced teeth, RED proportion, minimal prep, ceramic veneers
Volume 12 Issue 2 - 202I

\author{
Haifa Barakah \\ King Saud University, Saudi Arabia
}

Correspondence: Haifa Barakah, King Saud University, P.o. box 426 I5, Riyadh, I I55I, Saudi Arabia, Tel +966504426343,

Email hbarakah@ksu.edu.sa

Received: March 29, 202I | Published: May 14, 202 |

\section{Abbreviations: RED, recurring esthetic dental}

\section{Introduction}

Esthetics is the science of harmonizing a restorative treatment with nature along with restoring shape and function of the teeth; meanwhile, reestablishing a new smile adapting to the patient's life style, job, social position as well as highlighting the esthetical features. ${ }^{1}$ The demand for esthetic dentistry has recently increased among patients willing to have a visually pleasing smile. Improved materials, restorative techniques, esthetic parameters, and the concept of preserving the remaining tooth structure favors the obtainment of healthy, functional and esthetical smiles. ${ }^{2}$

Ceramic veneers have been used as a successful approach to treat esthetic problems in anterior region due to its unique ability to reproduces the optical properties of the enamel and dentine such as fluorescence, opalescence, and translucency, as well as desirable intrinsic features as biocompatibility, high compressive strength, abrasion resistance, and color stability. ${ }^{3}$ Tooth preparation for ceramic veneer involves the removal of less than half the thickness of the enamel, preserving the tooth structure where a reliable bonding of the ceramic veneers to the rest of intact enamel. ${ }^{4}$

A dentition with spaces is considered a normal type of occlusion, which is found in almost one third of the population. ${ }^{5}$ According to the epidemiological study of Steigman and Weissberg (1985), 21.4\% of the general population presented spacing in both arches, whereas $50 \%$ of people with spacing had spaces in both arches. ${ }^{6}$ The causes of spacing may be hereditary, acquired or functional. Hereditary causes include tooth size-arch size discrepancies, congenitally missing teeth, macroglossia, supernumerary teeth, small teeth and hypertrophic upperlip frenum. Functional causes include deleterious oral habits, whereas acquired causes include pathologic conditions such as increasing tongue size, missing teeth, delayed eruption of permanent teeth and periodontal disease. Certain spacing cases are well accepted by patients and treatment is not necessary. However, in cases needing treatment, the therapeutic options may include simple esthetic composite resins, orthodontic space closure, or both. ${ }^{7}$

In the cases of jaw size-teeth size discrepancy, a careful diagnosis and treatment plan are the key factors for successful results that can address the patient's needs. The aim of this paper is to report the treatment of a patient with aesthetic concerns in the anterior teeth despite receiving an orthodontic treatment, using RED proportion with minimally invasive restorative approach and evaluating the clinical performance after three years.

\section{Case description}

\section{Diagnosis and treatment planning}

A 45 years old female presented to the dental restorative clinic in the dental collage of King Saud University in Riyadh, Saudi Arabia, for enhancing the appearance of her anterior teeth. The patient was hypertensive controlled with medication for more than five years. Her main concern was to close the spaces between her teeth in the maxillary and mandibular anterior regions. The patient stated that she had an orthodontic treatment to correct her main concern ten years ago then she had a permanent retainers after the treatment. During that time, she noticed the unpleasant appearance of her anterior teeth with retro-clined appearance associated with persistent gingival inflammation despite having periodontal consultation and treatment. After a while, she had that retainers removed and accordingly, the teeth returned to the original spaced position. In the present restorative clinic, a thorough clinical and radiographical examination were performed. Photographic data was collected from the patient, alginate impressions (United Alginate, Major Prodotti Dentari SpA, Moncalieri, Italy) were taken for diagnostic models.

The following findings were noticed (Figure 1):

a) Generalized spaces between the teeth anteriorly in the maxillary and mandibular jaws. The spaces were seemed to be as a result of jaws-teeth size discrepancy which was mostly hereditary in origin.

b) Mild marginal-rolled gingivitis around the anterior teeth.

c) The appearance of short anterior clinical crowns caused by undersized teeth.

d) Disharmony in the relation of the incisal curvature of the upper incisors to the lower lip

e) Short clinical canines. 

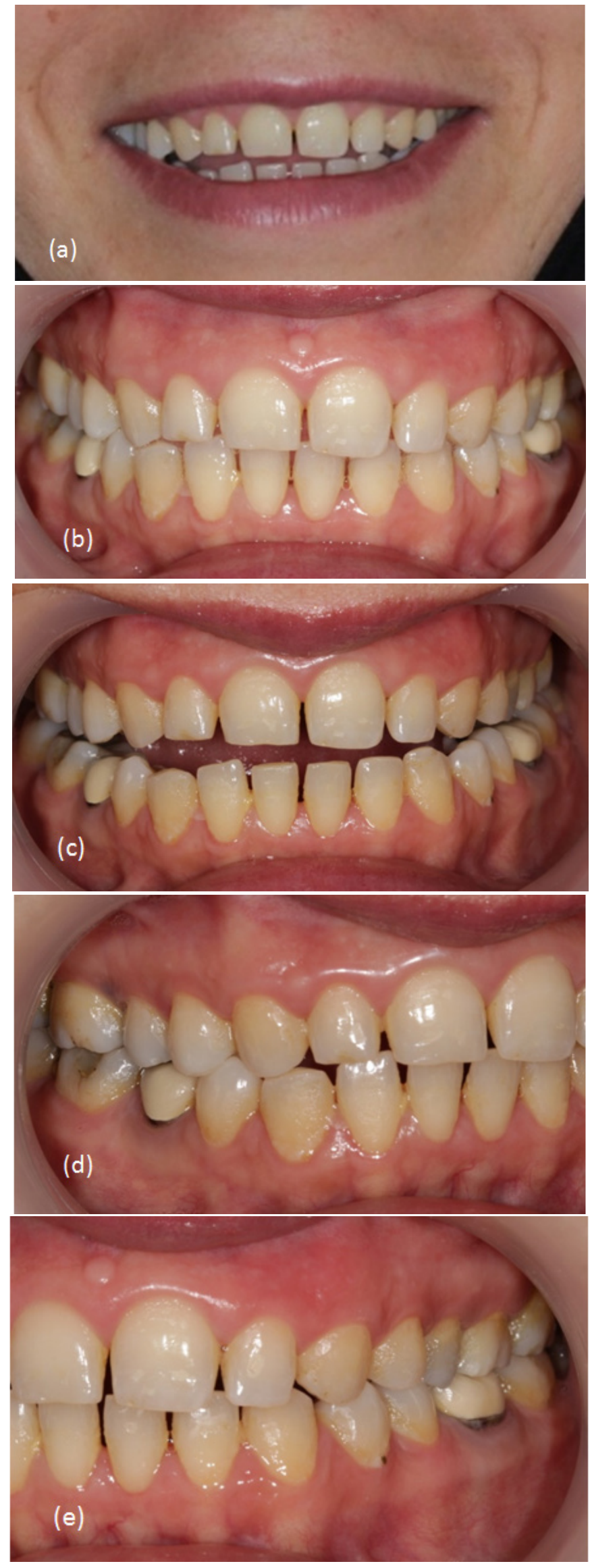

Citation: Barakah H. Esthetic ceramic veneers for spaced anterior teeth utilizing the RED proportion; a case report with three years follow-up.J Dent Health Oral Disord Ther. 2021;12(2):46-50. DOI: 10.15406/jdhodt.202I.12.00548
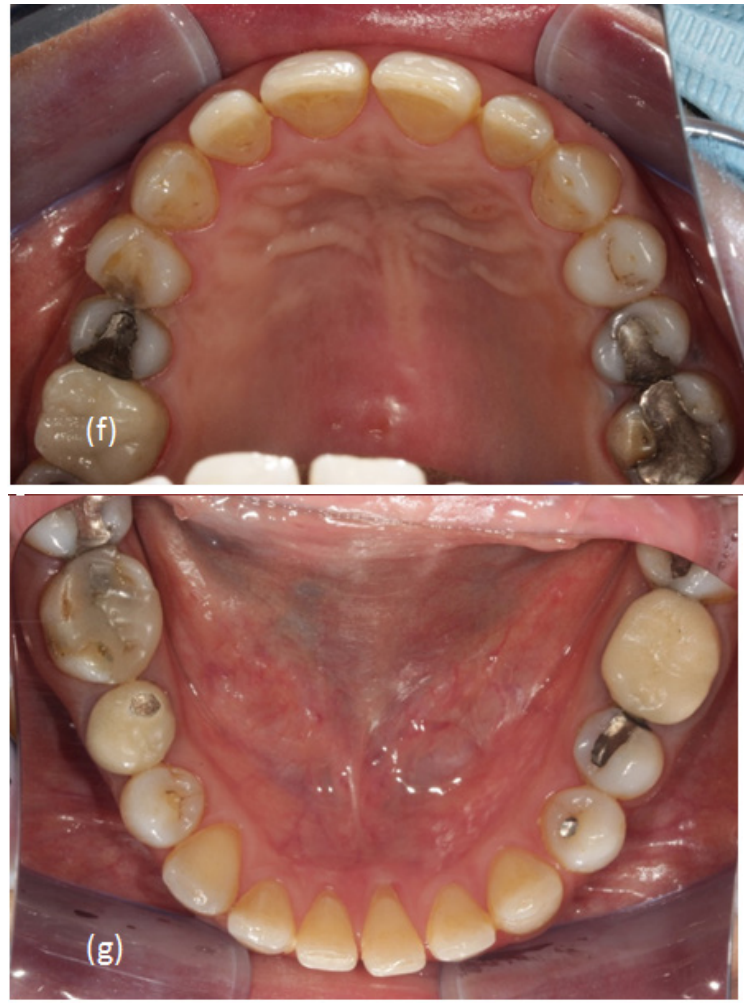

Figure I (a) Lip position during a smile, smile line, and teeth visibility. (b) Lip position of the teeth in occlusion. (c) Appearance of teeth in slight-open position. (d) Right view. (e) Left view. (f) occlusal view of the maxillary teeth. (g) Occlusal view of mandibular teeth.

The first stage of the restorative procedure was mounting the diagnostic models on a semi-adjustable articulator. They were studied to decide the shape and size of the restorations using Recurring Esthetic Dental (RED) Proportion which has been proposed as a model in designing smiles. Its concept is based on several related factors of variability including the tooth, face, and body into the calculations. The RED proportion states that the proportion of the successive widths of the maxillary teeth as viewed from the front should remain constant, progressing distally. The frontal view width of every maxillary tooth becomes smaller by a certain percentage as one moves posteriorly. ${ }^{8,9}$

It was reported by Ward DH. (2008), that the width of the lateral incisor is reduced by a selected percentage from the width of the central incisor, and the width of each tooth distally is reduced by this same percentage from its mesial tooth. The $70 \%$ RED proportion has been recommended for normal-length teeth with a $78 \%$ width/ height ratio of the maxillary central incisors that coordinates with the body/tooth height. ${ }^{8,9}$ This proportion came along with the facial and dental criteria of our patient (Figure $2 \& 3$ ). When using the $70 \%$ RED proportion, assuming the width of the central incisor is (X). (X) can be obtained from the formula dividing the inter-canines width with a constant ICW/4.38 (Adapted from Ward DH. 2008). ${ }^{9}$

From the diagnostic cast (Figure 3), the ICW was found to be $40 \mathrm{~mm}$.

$$
\begin{array}{r}
X=I C W / 4.38=40 \mathrm{~mm} / 4.38=9.1 \mathrm{~mm} \\
\Rightarrow(\text { Central Incisor Width }) C I W=9.1 \mathrm{~mm}
\end{array}
$$

The width of the maxillary lateral incisor (LIW) is $70 \%$ of the central incisor width, which means: 


$$
L I W=0.7 C I W=0.7 * 9.1=6.37 \mathrm{~mm}
$$

Accordingly, the canine width $(\mathrm{CW})$ is:

$$
C W=0.7 * 0.7 * C I W=0.49 * 9.1=4.46 \mathrm{~mm}
$$

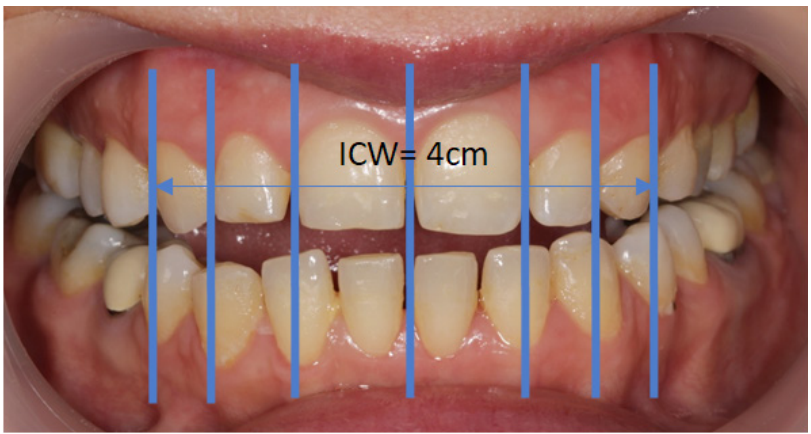

Figure 2 Inter-canine width ICW.

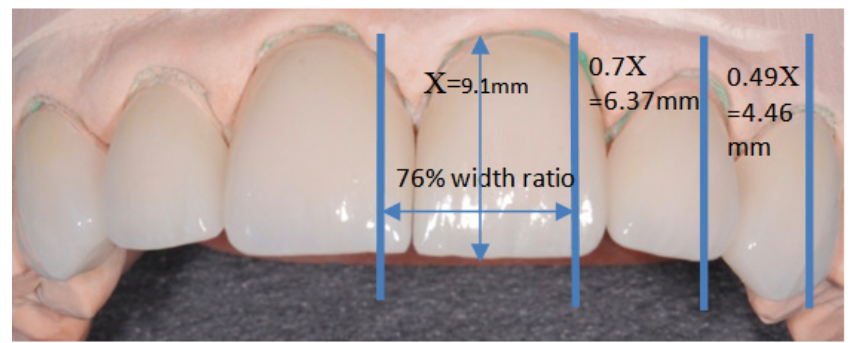

Figure 3 RED Proportion (70\% for Normal length teeth).

In an esthetic smile, the proportion or ratio between the central and lateral incisor should be constant, progressing anteriorly to posteriorly in the mouth. ${ }^{9}$

The diagnostic wax-up was then presented to the patient with a simulation or mock-up using a transparent template with a restorative material defining the choice of color, shape, width and length of anterior upper incisors and canines to develop a curvature harmonized with the upper border of the lower lip (Figure 1a). The patient was provided with the option of ceramic veneers for the maxillary and mandibular anterior teeth. The final treatment plan was approved.

\section{Treatment stages}

\section{A. Periodontal stage}

Gingivectomy and gingivo-plasty using a water-lase Surgical laser (WaterLase ${ }^{\circledR}$ iPlus, dentalEr,Cr:YSGG trolley-mounted) to adjust the gingival contour and the length of the upper anterior teeth according to RED proportion as shown in Figure 4. Then the patient was seen one week later for monitoring the healing process of the gingivae which seemed to be in a good condition (Figure 5).

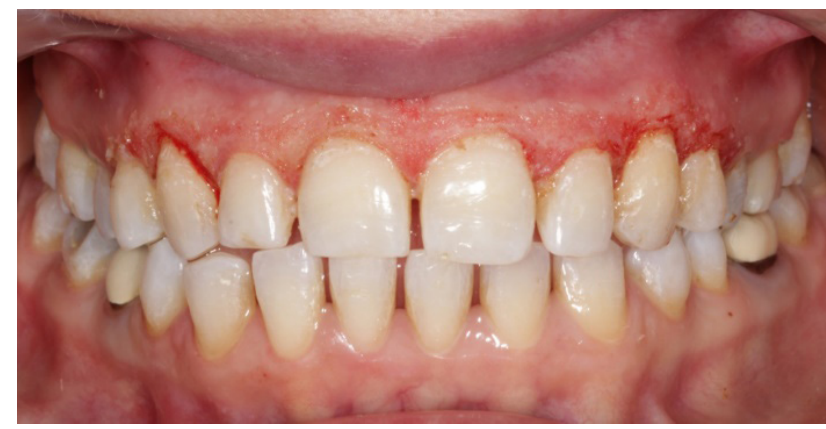

Figure 4 Gingivectomy and gingivoplasty of the maxillary anterior teeth.

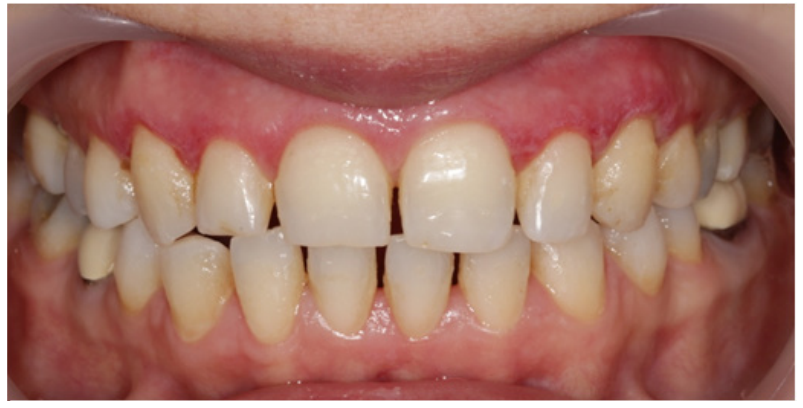

Figure 5 One week after gingevectomy.

\section{B. Preparation and impression}

Fabrication of a diagnostic mock-up using the previously fabricated template and a temporary restorative material restoration where the preparation was performed based on to be as a reference for the amount of preparation needed. A conservative A $0.3 \mathrm{~mm}$ shallow chamfer finish line was prepared on the maxillary and mandibular anterior teeth for the ceramic veneers using the depth cutting diamond bur (DIATECH, Dübendorf, Switzerland). The surface was then smoothened using the long tapered coarse diamond bur followed by the fine one (DIATECH, Dübendorf, Switzerland) as shown in Figure 6. At the same appointment, gingival displacement was obtained using retraction cords (\#00, Ultradent, South Jordan UT, USA) and the final impression was done, using silicone impression material (Aquasil impression material, Dentsply, International Inc. Canada). The ceramic shade was designated as A1. The glass-ceramic lithium disilicate was used (IPS e.max Press, Ivoclar Vivadent Schaan, Liechtenstein, Zurich). Temporary veneers were then fabricated using the transparent template and Protemp 4 provisional material (3M ESPE St. Paul, MN, and USA). The provisional veneers were cemented with Temp-Bond NE (Kerr, Orange, CA) temporary cement as shown in Figure 7.

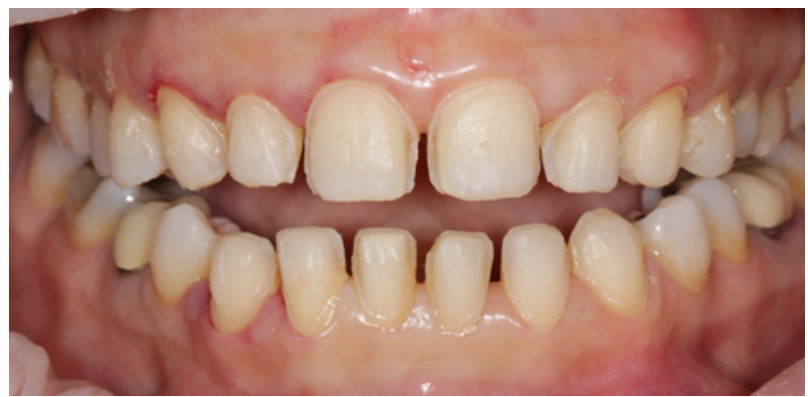

Figure 6 Veneer preparations of the maxillary and mandibular anterior teeth

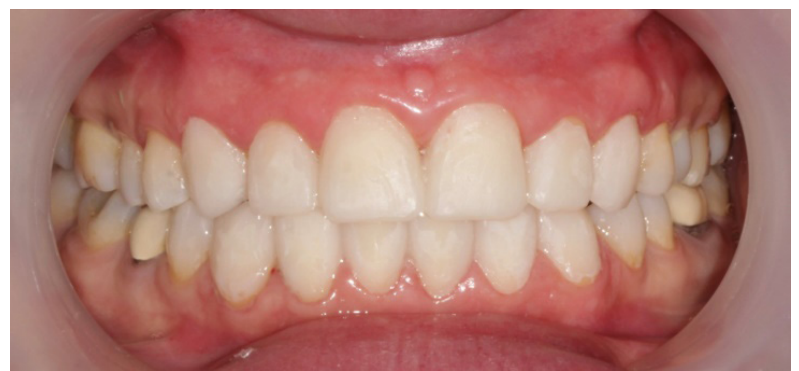

Figure 7 Temporization using a temporary restorative material.

\section{Veneers try-in and cementation}

The prepared teeth were cleaned and the veneers were tried-in. The marginal adaptation, proximal contacts, shade and alignment 
of the veneers as well as the periodontal relation were checked. The cementation was done using dual cure resin cement Veriolink Esthetics DC (Ivoclar Vivadent, Schaan, Lichtenstein). The internal surfaces of the veneers were cleaned with Ivoclean (Ivoclar Vivadent, Schaan, Lichtenstein) and then etched with 9.5\% hydrofluoric acid for 30 seconds (Condicionador de Porcelanas, Dentsply Brasil). The surfaces were washed with water, and dried with an air syringe. The veneers were silanized with a silane coupling agent (Monobond Plus, Ivoclar Vivadent) that was applied and left to react for $60 \mathrm{~s}$ before air drying. The enamel was conditioned with $37 \%$ phosphoric acid for 30seconds (Total Etch, Ivoclar Vivadent, Schaan, Lichtenstein) then washed for another 30s and gently dried. The adhesive agent (ExciTE F DSC, Ivoclar Vivadent, Schaan, and Lichtenstein)) was applied on both the enamel (for 10s using a micro-brush) and the inner surface of the veneers. Excess bonding was blown to a thin film using air spray. Then the cement was applied on the inner surface of the veneers that were then seated on the teeth. The cementation procedure was performed one-by-one starting with the maxillary central incisors, laterals, and canines. Then the same sequence was followed on the mandibular anterior teeth. Excess cement was removed with a brush, and each surface was photo activated for 60seconds using LEDcuring unit (Blue phase LED-curing light, Ivoclar Vivadent, Schaan, Lichtenstein).It was then removed after curing using a \#12 surgical blade. Flossing was performed at the proximal areas to ensure patent interproximal contacts. The restorations margins were finished and polished using ultrafine diamond tapered bur (DIATECH, Dübendorf, Switzerland).

\section{Follow-up and recall appointments}

The patient was seen one week later to the cementation visit for checking of any remaining excess cement or occlusal adjustments. Then the patient was seen again three years later for a recall appointment. The veneers seemed to be in a good condition with a healthy gingivae except for slight plaque accumulation on the cervical margins of the mandibular anterior teeth (Figure 10\&11).

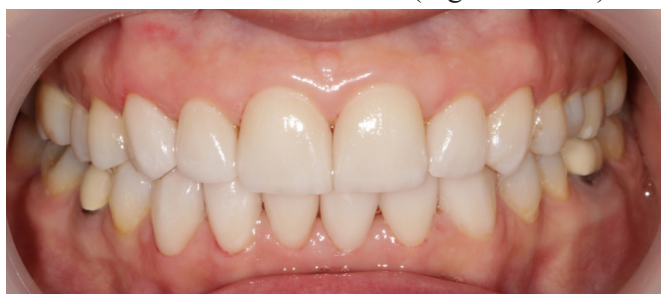

Figure 8 Porcelain veneers after cementation.

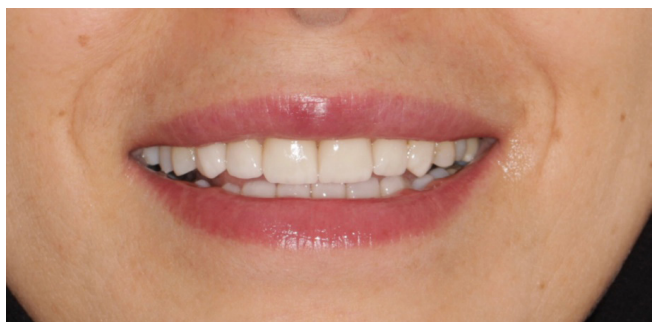

Figure 9 Smile view after veneer cementation.

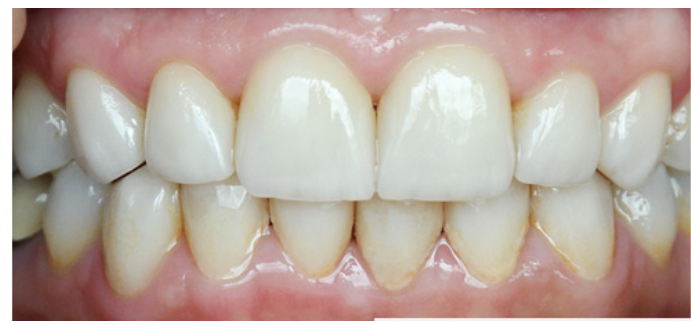

Figure 10 Front view three-years post-op.

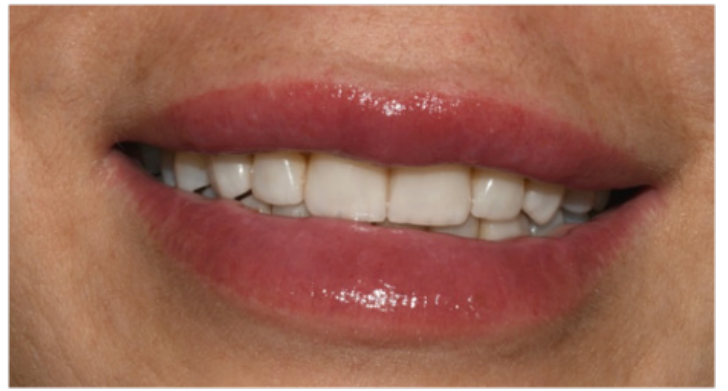

Figure I I Smile view three-years post-op.

\section{Discussion}

There are several treatment options to be considered for multiple spaces between the teeth in the anterior regions. The treatment could be orthodontic, restorative or both. ${ }^{10}$ In the present case, the patient had an orthodontic treatment ten years prior to attending to the restorative clinic asking to close her anterior spaces. Since there is a discrepancy in jaw-teeth sizes where the teeth are smaller than the available jaw space, orthodontic treatment had failed to solve the problem. Multidisciplinary treatment of orthodontic alignment of the teeth and equally re-distribute the spaces, then reshape the small teeth by a restorative mean of treatment was considered the appropriate approach.

A treatment plan is necessary as an aid for communicating to the patient what is required procedures of treatment. It also explains the sequence of treatment involved. A diagnostic wax-up is an initial visualization tool useful in representing the desired final outcome of the treatment.

To reshape spaced small teeth, porcelain laminate veneers are more esthetic than direct or indirect composite veneers and are also considered to be a conservative approach due to the small amount of enamel reduction on the labial surface. When comparing direct composite veneers to porcelain veneers it was reported a higher risk of failure at a 2.5 -year evaluation. ${ }^{11}$ The survival rate of 182 porcelain laminate veneers that were bonded using correct adhesive techniques was found to be $94.4 \%$ at 12 years. ${ }^{12}$ Another study revealed only $4 \%$ of 87 veneers having to be replaced at follow-up which represented an excellent retention rate of porcelain laminate veneers after 10 years. The $4 \%$ of noted failures were found to possess microleakage, marginal discoloration, and fractures. ${ }^{13}$ Good patient instruction and regular professional follow-up and are the keys to ensure the longevity of porcelain laminate veneers. Our patient was instructed to regularly floss after routine toothbrushing and to attend the follow-up checkup dental visits and a careful scaling and prophylaxis. She was also advised to avoid chewing hard substances such as candies, ice, etc.

\section{Conclusion}

Thorough case analysis, carful treatment plan with the use of RED proportion and maintaining good oral hygiene had contribute to the treatment success that meet the patient esthetic expectation.

\section{Acknowledgments}

None.

\section{Conflicts of interest}

I wish to confirm that there are no known conflicts of interest associated with this publication and there has been no significant financial support for this work that could have influenced its outcome. 


\section{Funding}

None.

\section{References}

1. Venâncio, Gisely Naura, Rodrigues Guimarães, et al. Conservative esthetic solution with ceramic laminates: literature review. RSBO Revista Sul-Brasileira de Odontologia [en linea]. 2014;11(2):185-191.

2. Walter RD, Raigrodski AJ. Critical appraisal: clinical considerations for restoring mandibular incisors with porcelain laminate veneers. $J$ Esthet Restor Dent. 2008;20(4):276-281.

3. Pini NP, Aguiar FH, Lima DA, et al. Advances in dental veneers: materials, applications, and techniques. Clin Cosmet Investig Dent. 2012;4:9-16.

4. Steigman S, Weissberg Y. Spaced dentition. An epidemiologic study. Angle Orthod. 1985;55(2):167-167.

5. Steigman S, Gershkovitz E, Harari D. Characteristics and stability of spaced dentition. Angle Orthod. 1985;55(4):321-328.
6. Gkantidis N, Psomiadis S, Topouzelis N. Teeth spacing: Etiology and treatment. Hell Orthod Rev. 2007;10:75-92.

7. Ward DH. Using the RED proportion to engineer the perfect smile. Dent Today. 2008;27(5):116.

8. Ward DH. Proportional smile design using the recurring esthetic dental (red) proportion. Dent Clin North Am. 2001;45(1):143-154.

9. Ittipuriphat I, Leevailoj C. Anterior space management: interdisciplinary concepts. J Esthet Restor Dent. 2013;25(1):16-30.

10. Meijering AC, Creugers NHJ, Roeters FJM, et al. Survival of three types of veneer restorations in a clinical trial: a 2.5 -year interim evaluation. $J$ Dent. 1998;26(7):563-568.

11. Fradeani M, Redemagni M, Corrado M. Porcelain laminate veneers: 6to $12-$ year clinical evaluation - a retrospective study. Int J Periodontics Restorative Dent. 2005;25(1):9-17.

12. Peumans M, De Munck J, Fieuws S, et al. A prospective ten-year clinical trial of porcelain veneers. $J$ Adhes Dent. 2004;6(1):65-76. 